\title{
PAPER
}

\section{Application of time-frequency analysis to somatosensory evoked potential for intraoperative spinal cord monitoring}

\author{
Y Hu, K D K Luk, W W Lu, J C Y Leong
}

See end of article for authors' affiliations

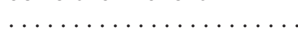

Correspondence to: Dr Y Hu, Duchess of Kent Children's Hospital, Department of Orthopaedic Surgery, The University of Hong Kong, Hong Kong; yhud@hkusua.hku.hk

Received 15 April 2002 In revised form 17 September 2002 Accepted 19 September 2002

\begin{abstract}
Objective: To investigate the improvement in the reliability of intraoperative spinal cord monitoring by applying time-frequency analysis to somatosensory evoked potentials (SEP).

Methods: 34 patients undergoing scoliosis surgery were studied. SEP were recorded during different stages of scoliosis surgery. Averaged SEP signals were analysed intraoperatively by short time Fourier transform (STFT). The time-frequency characteristics of SEP were observed during surgery. The main peak in the time-frequency interpretation of SEP was measured in peak time, peak frequency, and peak power. The changes in these variables were compared with the changes in latency and amplitude during different surgical stages.

Results: During different surgical stages, changes in peak times and peak powers were found to correlate with the changes in latency and amplitude, respectively. Peak time showed more variability than latency $(p<0.01)$, while peak power showed less variability than amplitude $(p<0.01)$. The peak frequency of SEP appeared to be unchanged during surgery. SEP signals were found to have specific time-frequency characteristics, with the time-frequency distribution of the signals being located in a particular time-frequency space.

Conclusions: Time-frequency analysis of SEP waveforms reveals stable and easily identifiable characteristics. Peak power is recommended as a more reliable monitoring parameter than amplitude, while peak time monitoring was not superior to latency measurement. Applying time-frequency analysis to SEP can improve the reliability of intraoperative spinal cord monitoring.
\end{abstract}

$\mathrm{S}$ pinal surgery is an effective way of correcting deformities of the spine, but also entails the risk of damage to the spinal cord. The use of spinal cord monitoring can minimise such risks and has become a widely used technique in conjunction with spinal surgery. ${ }^{1}$ Previous studies have shown that changes in somatosensory evoked potential (SEP) can indicate neurological deficits in the spinal cord. ${ }^{23}$ Intraoperative SEP monitoring can therefore provide routine monitoring of the functional status of the cord. The technique has become one of the most popular clinical tools in identifying impairment of the cord during spinal surgery. ${ }^{2}$

Current SEP monitoring techniques measure the amplitude and latency of the waveform to quantify any changes in SEP and to detect injury to the spinal cord. This monitoring procedure is based on time domain detection. However, SEP signals recorded in the operating theatre are usually accompanied by noise, ${ }^{2}$ which increases signal variability, makes peak identification difficult, and often precludes accurate latency and amplitude measurements. ${ }^{34}$

Frequency analysis can simplify the understanding of the waveform. Ryan and Britt ${ }^{5}$ applied fast Fourier transform (FFT) analysis to intraoperative SEP monitoring and found that the power spectra of the SEPs remained stable during surgery. Previous studies ${ }^{5-9}$ have proved that deficits in spinal cord function cause localised changes (that is, changes occurring at various time instances or delays) in the morphology and therefore in the spectrum of the SEP waveform, ${ }^{7}$ suggesting that the frequency (or spectral) changes occurring in the SEP may be an important indicator of spinal cord injury. ${ }^{8} 9$ However, the main disadvantage of power spectra is the loss of time information. In order to obtain information on the signal energy at a specific time as well as frequency, time-frequency analysis methods must be used.
Time-frequency analysis can provide both temporal and spectral information on the SEP waveform, which allows computation of parameters in the combined time and frequency domain. ${ }^{910}$ Time-frequency analysis of SEP waveforms could therefore provide an improved technique for detecting neurological injury. Previous experimental studies have suggested the value of time-frequency analysis in detecting spinal cord injury, and have shown that it may be a good method for analysing the SEP waveform. ${ }^{79}$ However, the application of time-frequency analysis to intraoperative SEP monitoring has not yet been reported.

Our aim in this study was to apply time-frequency analysis to SEP signals recorded during surgery to investigate their role in improving the reliability of intraoperative spinal cord monitoring. We observed the time-frequency distribution of SEP signals during surgery and measured variables in both time domain and time-frequency domain that may be used as diagnostic indicators of injury. The changes in time-frequency analysis indiced at different surgical stages were examined, and the value of time-frequency analysis during SEP monitoring in clinical practice was evaluated by comparison with conventional peak latency-amplitude analysis techniques.

\section{METHODS}

Thirty four patients undergoing scoliosis surgery were included in the study (27 female and seven male). Their age ranged from 11 to 19 years (mean 15 years).

All patients received general anaesthesia, induced by thiopentone (thiopental) $(0.4 \mathrm{mg} / \mathrm{kg})$ or fentanyl ( $1-2 \mu \mathrm{g} / \mathrm{kg})$.

Abbreviations: FFT, fast Fourier transform; SEP, somatosensory evoked potential; STFT, short time Fourier transform 
Isoflurane $(0.5-0.8 \%)$ and nitrous oxide/oxygen (typically $60: 40 \%$ ) were used to maintain the anaesthesia. None of the patients had any neurological deficit before, immediately after, or at the two months assessment following the surgery.

To elicit SEP, a pair of stimulating electrodes was applied over the posterior tibial nerve behind the medial malleoli, with constant current stimulation in the range of 10 to $30 \mathrm{~mA}$. Single pulse stimulation with a frequency between 5.1 and $5.7 \mathrm{~Hz}$ and a duration of $300 \mu$ s was applied. The SEP signals were collected over $\mathrm{Cz}^{\prime}$ ( $2 \mathrm{~cm}$ posterior to $\mathrm{Cz}, 10-20$ international system of EEG electrode placement), and CV (on the cervical spine over the $\mathrm{C} 2$ process), versus the Fz of the 10-20 system, using subcutaneous needle electrodes.

An intraoperative spinal cord monitoring system (Nicolet Viking IV, Nicolet Biomedical, Madison, Wisconsin, USA) was employed to record the responses, with noise reduction achieved using a 20-3000 $\mathrm{Hz}$ bandpass filter and automatic artefact rejection. The sweep time of SEP recording was 100 ms. Continuous averaging was used, with 100 times averaging. The initial negative and positive waves were identified in the SEP tracings. Averaged SEP signals were then analysed intraoperatively on a personal computer (IBM 380XD) using Labview 5.0 software. During surgery, surface electric stimulation was applied to the posterior tibial nerve on both sides. The averaged SEP were measured alternately during left or right sided stimulation every 15 to 20 minutes during non-critical procedures such as incision, and continuously during critical surgical procedures or if abnormal SEP occurred.

Our previous study showed that short time Fourier transform (STFT) time-frequency analysis is the best algorithm for time-frequency analysis of SEP signals. ${ }^{11}$ This method breaks up the signal into small time intervals and uses Fourier analysis on each time interval. For a recorded SEP signal $s(t)$, the STFT spectrogram is defined by the following equation:

$$
\begin{aligned}
& \operatorname{STFT}_{\left.(t, \omega)\right|_{t=n \Delta t, \omega=\frac{2 k \pi}{N \Delta t}}}=\operatorname{STFT}(n, k) \\
& =\sum_{i=0}^{N-1} s(i) w(i-n) e^{-\frac{j 2 k i \pi}{N}}
\end{aligned}
$$

where $\mathrm{w}(\mathrm{n})$ is the window function, $\Delta \mathrm{t}$ denotes the time sampling interval, and $\mathrm{N}$ is the block length of the window function. In this study, a 20 points length Hanning window was used.

To compare the intervariability of SEP, both conventional amplitude/latency and time-frequency analysis parameters on the following surgical stages ${ }^{12}$ were analysed:

- Preoperation: once the patient had been anaesthetised and positioned on the operation table, the baseline was taken.

- Spine exposure: the second SEP was recorded when the muscle and soft tissues were stripped and the spine was exposed.

- Instrumentation loading: when the instrumentation-such as hooks, wires, or screws-was inserted. SEP was monitored continuously during this stage. The most reproducible response by visual interpretation of amplitude/ latency evaluation was used for analysis.

- Deformity correction: this is the stage when the deformity was corrected by rod rotation, compression, distraction, or tightening of sublaminar wires. Spinal monitoring was again done continuously and the most reproducible response was included for analysis.

- Wound closure: the stage when the wound was closed.

The latency and amplitude were measured from the SEP waveforms in the time domain, and the mean values and standard deviations calculated for the data collected from each patient. Percentage changes in amplitude and latency were calculated by comparing the values of latency and amplitude against those of baseline. Criteria for indicating possible spinal cord injury were a $10 \%$ increase in latency or a $50 \%$ decrease in amplitude. If an abnormal SEP occurred, SEP monitoring would be repeated two or three times to verify the abnormality. If the SEP returned to normal in 15 minutes, the change was defined as transient and not indicating spinal cord injury. If the abnormal SEP was persistent, the surgical procedure was halted, changes that could have affected the SEP signal (anaesthetic level, blood pressure, core temperature, and so on) were excluded, and if necessary, the correction applied to the spinal deformity was reduced or the implant removed.

In addition to the time domain parameters, the peak time, peak frequency, and peak power of the energy peak in the time-frequency distribution were also computed. To avoid the influence of late components in SEP, which may be very variable in intraoperative monitoring, we only measured the time-frequency distribution of the SEP waveform within 5-80 ms duration of $\mathrm{Cz}-\mathrm{Fz}$ recordings and within 5-50 ms of $\mathrm{Cv}-\mathrm{Fz}$ recordings. The percentage change in these time-frequency parameters was also calculated. A 10\% increase in peak time was used as the criterion for detecting neurological deficit, and $30 \%, 50 \%$, and $70 \%$ decreases in peak power were evaluated as further criteria of potential spinal cord injury during intraoperative spinal cord monitoring.

The within patient variability of various parameters was calculated from the ratio of deviation to the mean value, which was defined as:

Variability $=\mathrm{SD} /$ mean $\times 100 \%$

Comparisons between the time and time-frequency parameters were analysed using the paired two sample Student $t$ test. The changes among different surgical stages were analysed using one way parametric analysis of variance (ANOVA).

\section{RESULTS}

Figure 1 shows a sample of time-frequency analysis of SEP recorded during different surgical stages. The time-frequency distribution of SEP is presented as a two dimensional plot, with the $\mathrm{x}$ axis as time, the $\mathrm{y}$ axis as frequency, and the intensity of the time-frequency relation in relative colour. Beneath each time-frequency plot is shown the time domain waveform of the SEP. The scale of the intensity index is plotted to the left of each time-frequency plot.

As fig 1 shows, STFT time-frequency analysis of SEP (Cz) appears as a peak in a certain space in the time-frequency distribution, around a midpoint of 42 (3.6) $\mathrm{ms}$ and $42(7.4) \mathrm{Hz}$ (mean (SD)). The peak for SEP $(\mathrm{CV})$ is centred at a frequency of 87 (9.1) Hz and a latency of 34 (3) ms (fig 2). As such, 20 to $330 \mathrm{~Hz}$ is usually an optimal frequency band for timefrequency representation, allowing the main peak of SEP to be identified easily. If there are no neurological problems, this peak will remain in the bounds of this space throughout surgery. The peak in time-frequency space is somewhat more easily identifiable than the waveform in the time domain. However, locating the central point of the peak is a little difficult because of the limitation on time resolution in the STFT algorithm. In fig 2A, the SEP waveform appears to overlap small noise signal waveforms. Such noise at the onset of SEP can interfere with the measurement of latency and amplitude, but this high frequency noise sitting on the SEP waveform shown in fig $2 \mathrm{~A}$ is effectively removed by frequency analysis. Monitoring SEP signals as a time-frequency distribution can therefore avoid some of the noise and improve the signal to noise ratio because of dislodgment of high frequency noise in time-frequency space. 

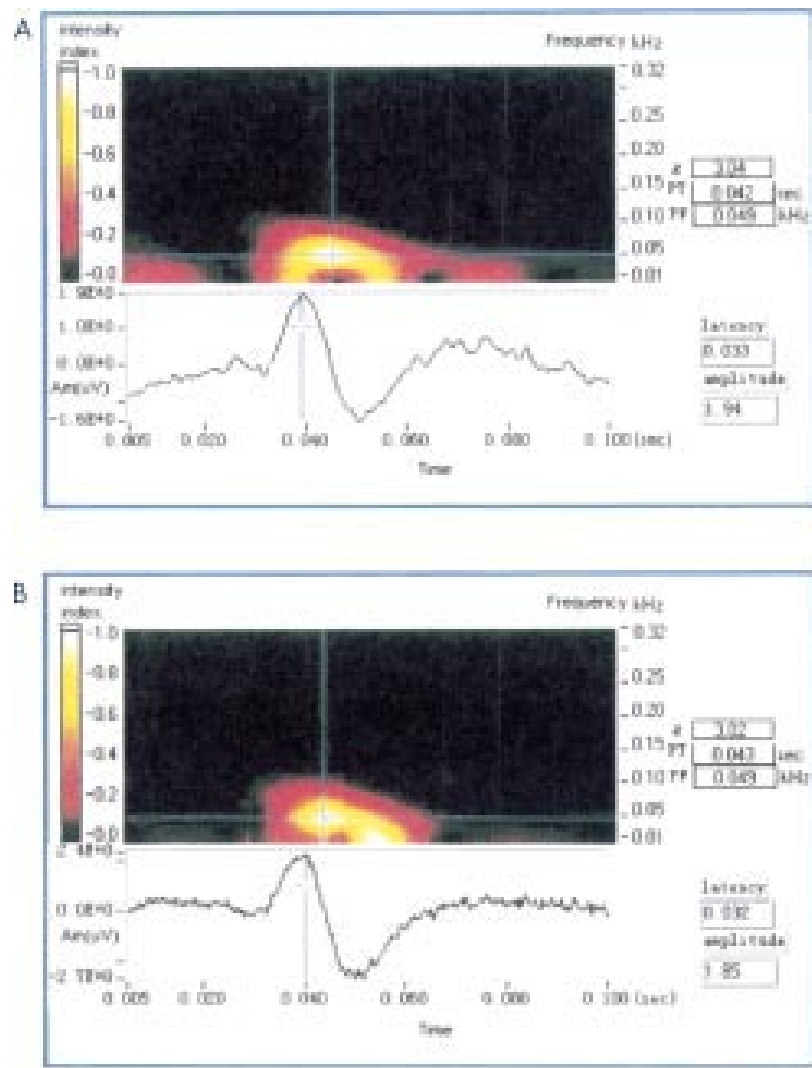

Figure 1 A sample of somatosensory evoked potentials (SEP) from $\mathrm{C} z^{\prime}-\mathrm{Fz}$ in a series of plots recorded at different stages of surgery: (A) preoperative; (B) spine exposure; (C) instrumentation loading; (D) deformity correction; and (E) wound closure. Each plot includes a time-frequency plot (upper graph) and a time domain P37/N45 waveform (lower graph). The initial negative and positive waves of the SEP were measured. On the right side of TFA graph, the time-frequency analysis values for peak power, peak time, and peak frequency are printed with the appropriate units labelled, while the values for latency and amplitude are similarly given to the right of the time domain waveform.

Three time-frequency variables (peak time, peak power, and peak frequency) of the SEP signals were measured during surgery (table 1). Table 1 compares the changes between time domain parameters and time-frequency parameters of the SEP at different surgical stages. Neither SEP latency mean values nor peak time mean values showed any significant changes over the five stages ( $p>0.05$ by ANOVA). In contrast, the SEP amplitudes showed significant differences at the different stages of surgery ( $\mathrm{p}<0.01$ by ANOVA), particularly between the first two stages. As table 1 shows, the SEP amplitude decreased from stage 1 to stage 2 , and then stayed fairly constant throughout stages 2, 3, and 4. Finally, the amplitude increased again in stage 5. Peak power of the SEP through these five surgical stages showed more stability than amplitude. Table 1 also shows the variability of peak frequency at the different surgical stages. No significant change in the peak frequency was seen during any of the stages $(\mathrm{p}<0.01$, by ANOVA).

To evaluate the criteria chosen as possible warning levels for monitoring, the changes in various parameters at different stages versus the baseline values were observed. One patient
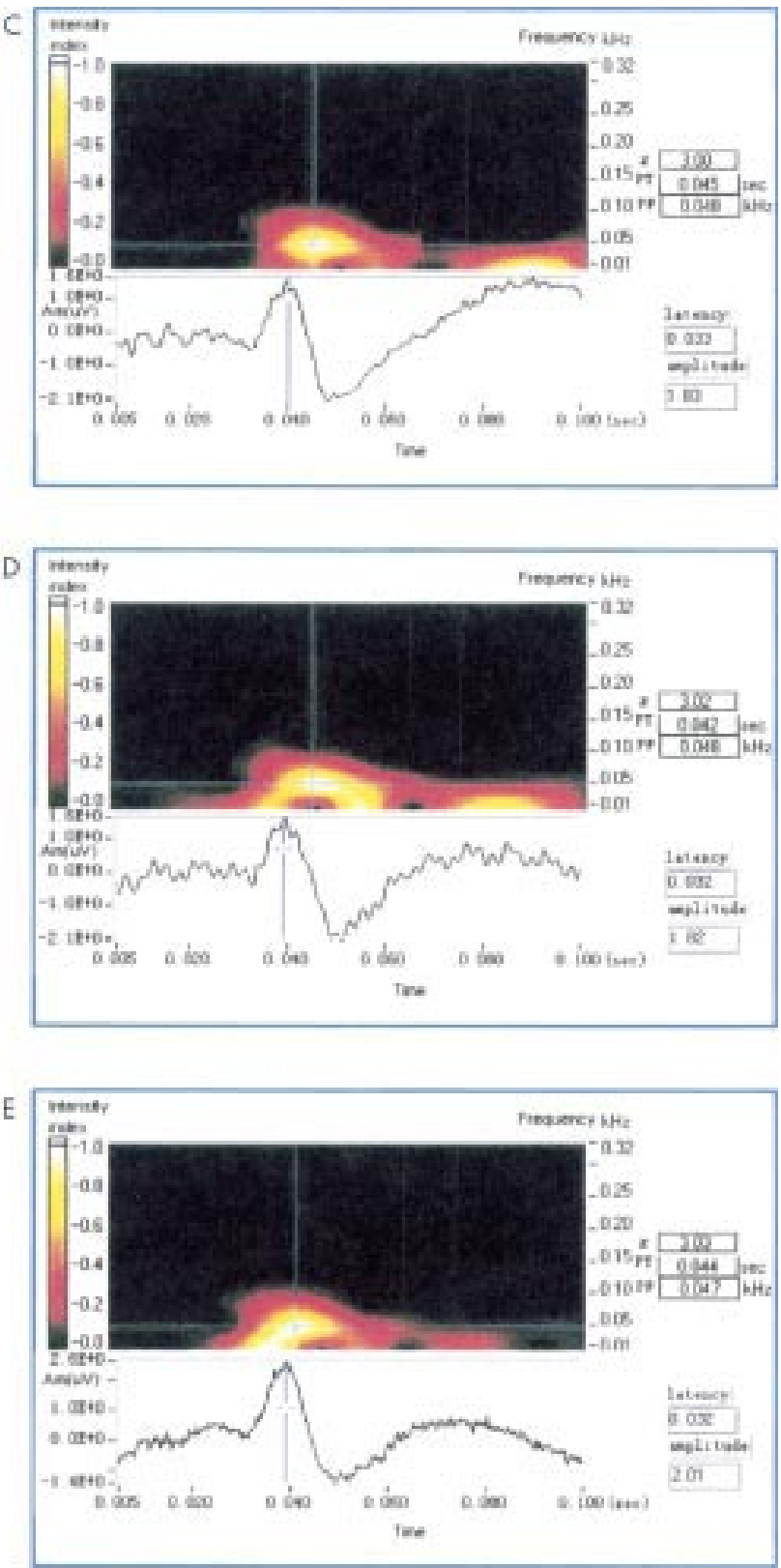

showed an abnormal latency increase in SEP (Cz) during left side stimulation as well as an abnormal peak time delay. Another patient showed an abnormal peak time delay in SEP (Cv). Four patients showed more than 50\% amplitude decrease in SEP $(\mathrm{Cz})$, while three showed more than 50\% amplitude decrease in SEP $(\mathrm{Cv})$. All the abnormal changes in SEP persisted for 45 minutes to 1.5 hours during surgery and were finally traced to a false positive output. However, the peak power of these patients remained stable and decreased to less than $50 \%$ of baseline, in spite of the lower SEP amplitude. Despite these abnormal SEP signals, none of the patients in this series showed any neurological deficit at any stage following surgery. Table 2 shows the specificity of spinal cord monitoring using the different parameters.

To evaluate the stability of various time domain and timefrequency domain parameters for intraoperative spinal cord monitoring, the within patient variability of these variables was calculated (table 3 ). Within patient variability of SEP latencies was relatively small, whereas that of the amplitudes was significantly larger. Peak time showed more variability than the latency $(\mathrm{p}<0.01$ by $t$ test); however, peak power 

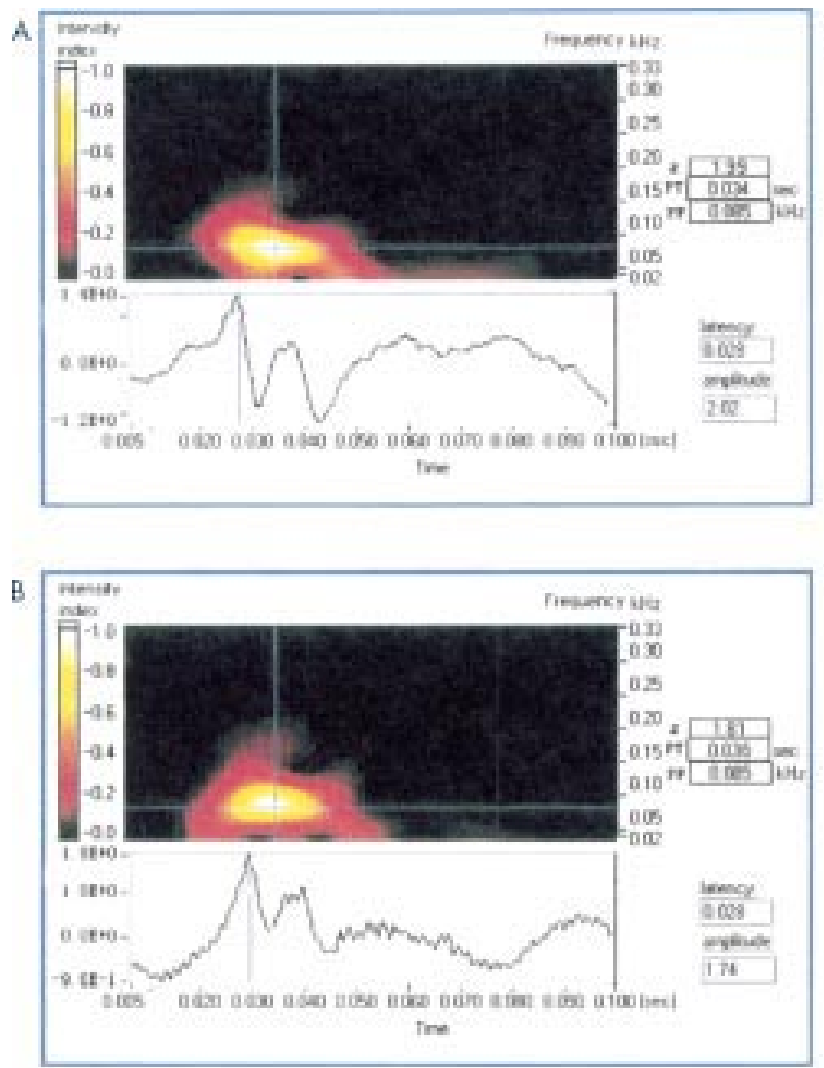

Figure 2 A sample of somatosensory evoked potentials (SEP) from $\mathrm{Cv}-\mathrm{Fz}$ in a series of plots recorded at different stages of surgery: $(\mathrm{A})$ preoperative; (B) spine exposure; (C) instrumentation loading; (D) deformity correction; and (E) wound closure. Each plot includes a time-frequency plot (upper graph) and a time domain N30/P35 waveform (lower graph). The initial negative and positive waves of the SEP were measured. On the right side of TFA graph, time-frequency analysis values for peak power, peak time, and peak frequency are printed with the appropriate units labelled, while the values for latency and amplitude are similarly given to the right of the time domain waveform.

showed significantly lower variability than amplitude $(\mathrm{p}<0.01$ by $t$ test $)$.

\section{DISCUSSION}

Hitherto, intraoperative SEP monitoring has mainly depended on time domain measurements of the latency and amplitude in the initial response peak. Under clinical conditions, SEP signals are usually accompanied by numerous noise signals from the patient and the environment. ${ }^{2}$ For example, the cortical peaks are often modulated by slow waves, which may result in apparent reductions or increases in amplitude, depending on the position of the peak relative to the ascending or descending part of the slow wave. Even with averaging signal enhancement, identification of SEP peaks is sometimes not an easy task and often requires trained personnel. ${ }^{16}$ False outcomes in SEP monitoring are probably caused by interpretation of technically unsatisfactory recordings. ${ }^{13}{ }^{14}$ Some false monitoring outcomes reflect contaminated SEP signals. ${ }^{3}$

Time-frequency analysis can separate the useful information from noise in time-frequency space so as to avoid the
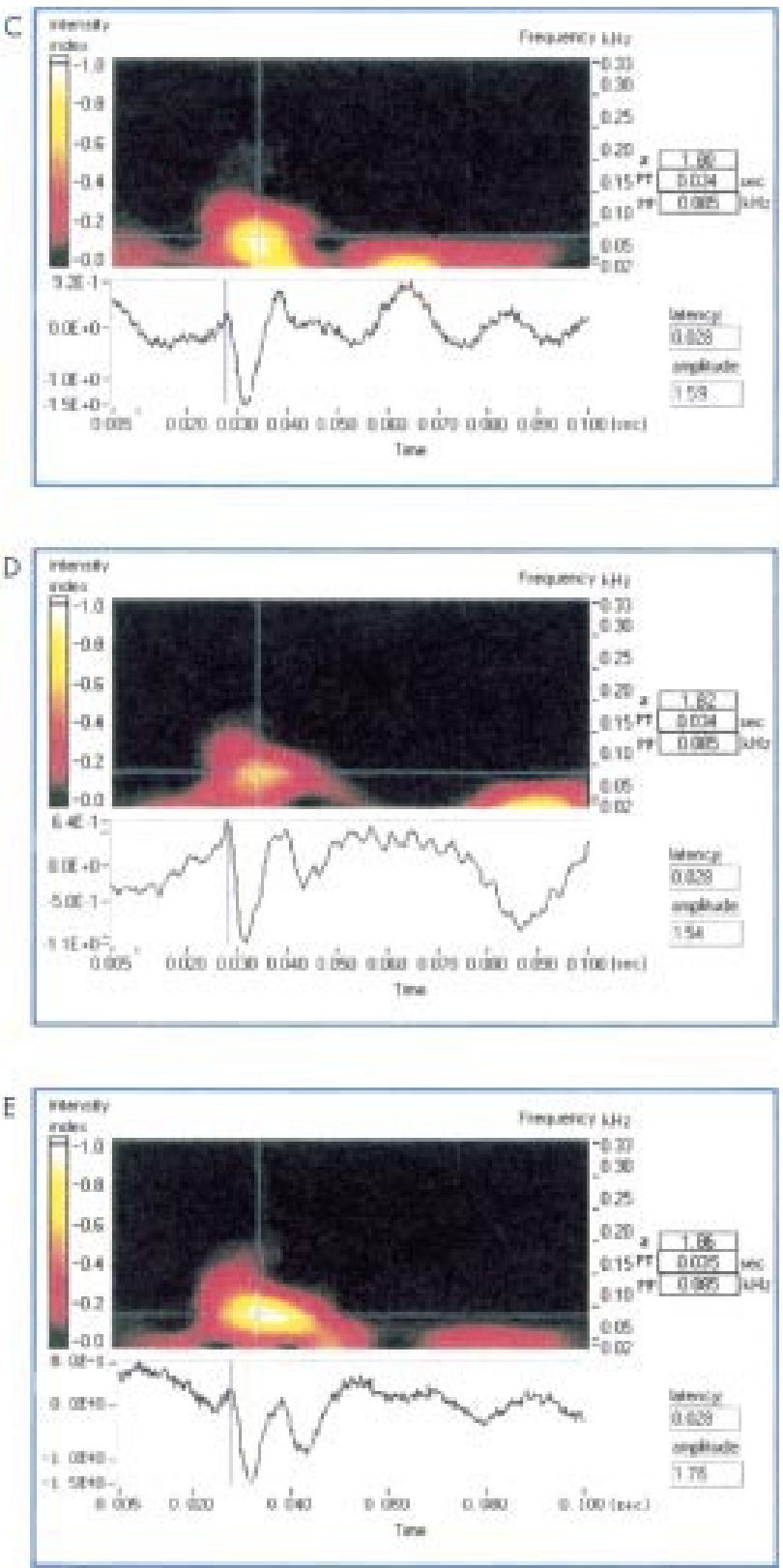

variability caused by noise. Our results show that the SEP signal presents as a single and definite component in timefrequency space. This time-frequency component was found to remain stable during surgery if there were no changes in neurological function. Identification of the SEP peak in timefrequency distributions is therefore simple and convenient. Peak power was found to be a much more stable variable for spinal cord monitoring than amplitude, which suggests that the former is a valuable adjunct to time domain amplitude measurement.

As a response to external stimulation, SEP reflects nervous activity along a certain pathway. ${ }^{15}$ Thus SEP signals consist of specific frequency components of finite magnitude which change with time. ${ }^{90}$ Previous studies have found that SEP signals consist of definite power spectra components which are located around a stable centre frequency. ${ }^{5111617}$ In our study, we found that normal SEP during surgery always appear at a distinct and stable time-frequency location.

Time-frequency analysis of SEP is also advantageous in that it represents the features of a complete waveform, not only the initial curve or peak. In addition to the usually 
Table 1 Variability of SEP amplitude/latency and time-frequency parameters in different surgical stages

\begin{tabular}{|c|c|c|c|c|c|c|c|}
\hline & & Stage 1 & Stage 2 & Stage 3 & Stage 4 & Stage 5 & Average \\
\hline \multicolumn{8}{|c|}{ (A) Variability of SEP amplitude/latency } \\
\hline \multirow[t]{2}{*}{ Amplitude } & SEP $(C z)$ & $1.2(0.46)$ & $0.95(0.47)$ & $0.92(0.41)$ & $1.03(0.55)$ & $1.13(0.70)$ & $0.99(0.38)$ \\
\hline & SEP (Cv) & $1.51(0.50)$ & $1.35(0.46)$ & $1.36(0.47)$ & $1.35(0.46)$ & $1.49(0.40)$ & $1.39(0.39)$ \\
\hline \multirow[t]{2}{*}{ Latency } & SEP (Cz) & $36.8(2.1)$ & $36.9(2.7)$ & $36.9(2.1)$ & $36.9(3.0)$ & $36.9(2.8)$ & $36.8(2.5)$ \\
\hline & SEP (Cv) & $26.6(1.7)$ & 26.7 (1.9) & $26.7(2.1)$ & $26.7(1.8)$ & $26.6(1.9)$ & $26.7(1.4)$ \\
\hline \multicolumn{8}{|c|}{ (B) Variability of time-frequency parameters } \\
\hline \multirow[t]{2}{*}{ Peak time } & $\mathrm{SEP}(\mathrm{Cz})$ & $40.8(1.3)$ & $40.8(3.1)$ & 40.8 (3.9) & 40.9 (4.1) & $40.8(2.8)$ & $40.8(1.6)$ \\
\hline & SEP (Cv) & $29.4(1.5)$ & 29.5 (1.8) & 29.5 (1.8) & 29.5 (1.8) & 29.5 (1.8) & 29.5 (1.5) \\
\hline \multirow[t]{2}{*}{ Peak power } & SEP (Cz) & $1.04(0.56)$ & $1.06(0.54)$ & $0.99(0.50)$ & $1.01(0.47)$ & $1.04(0.60)$ & $1.03(0.16)$ \\
\hline & SEP (CV) & $1.36(0.46)$ & $1.32(0.41)$ & $1.33(0.46)$ & $1.37(0.43)$ & $1.38(0.42)$ & $1.35(0.13)$ \\
\hline \multirow{2}{*}{$\begin{array}{l}\text { Peak } \\
\text { frequency }\end{array}$} & SEP (Cz) & $44.6(5.9)$ & $44.2(5.1)$ & $44.2(3.4)$ & $45.8(6.6)$ & $44.3(2.8)$ & $44.6(2.2)$ \\
\hline & SEP (Cv) & 81.5 (5.8) & $81.3(7.2)$ & $81.0(5.9)$ & $81.0(6.8)$ & $81.2(7.5)$ & $81.2(1.4)$ \\
\hline
\end{tabular}

Variables are given as mean (SD)

Stages: 1, preoperation; 2 , spine exposure; 3 , instrumentation loading (insertion of hooks, wires, screws,

etc); 4, deformity correction; 5, wound closure (see text for fuller description).

Table 2 Specificities of somatosensory evoked potential (SEP) monitoring by different parameter measurements

\begin{tabular}{lll}
\hline & \multicolumn{2}{l}{ Specificity } \\
\cline { 2 - 3 } Parameter & SEP (Cz) & SEP (Cv) \\
\hline Latency longer $>10 \%$ & $97 \%$ & $100 \%$ \\
Peak time longer $>10 \%$ & $97 \%$ & $97 \%$ \\
Amplitude decrease $>50 \%$ & $88 \%$ & $91 \%$ \\
Peak power decrease $>30 \%$ & $91 \%$ & $100 \%$ \\
Peak power decrease $>50 \%$ & $100 \%$ & $100 \%$ \\
Peak power decrease $>70 \%$ & $100 \%$ & $100 \%$ \\
\hline
\end{tabular}

Table 3 Within patient variability of time and frequency parameters of somatosensory evoked potentials (SEP)

\begin{tabular}{|c|c|c|c|c|c|}
\hline & \multicolumn{5}{|c|}{ Percentage variability } \\
\hline & Amplitud & Latency & $\begin{array}{l}\text { Peak } \\
\text { time }\end{array}$ & $\begin{array}{l}\text { Peak } \\
\text { power }\end{array}$ & $\begin{array}{l}\text { Peak } \\
\text { frequency }\end{array}$ \\
\hline $\operatorname{SEP}(C z)$ & $27.0 \%$ & $1.4 \%$ & $3.4 \%$ & $9.5 \%$ & $4.6 \%$ \\
\hline SEP (CV) & $21.0 \%$ & $1.3 \%$ & $2.9 \%$ & $9.5 \%$ & $1.7 \%$ \\
\hline
\end{tabular}

measured initial peak of the SEP signal, there are various subwaves that contain information on nervous system function. ${ }^{1618}{ }^{19}$ Rossini et al found that the subcomponents of SEP waveforms were well defined and stable on frequency analysis and concluded that this might be a clinically useful procedure. ${ }^{20}$ The use of the entire waveform in calculations of peak power may explain its stability in comparison with amplitude, which relies on the height of one peak in the waveform.

In this study, the three variables measured in the time-frequency domain were peak power, peak time, and peak frequency. The first two are in theory related to the time domain parameters of amplitude and latency. Peak frequency indicates the morphological changes in waveform. If the SEP waveform changes, the relative peak in the time-frequency distribution may shift in frequency. The results of this study do not show any trend in the peak frequency changes, but in previous studies it has been noted that peak frequency falls when spinal cord injury occurs. ${ }^{7}$ In our study we did not find a significant difference between the changes in peak time and latency ( $\mathrm{p}>0.05$ by $t$ test). However, the low time resolution in time-frequency analysis was a limitation preventing accurate peak time measurement. Peak power reflects the energy of the SEP signal, which is likely to be correlated with the level of neurological activity. The peak power is located in a certain time-frequency space, and as such will not be affected by noise outside that space. In contrast to amplitude measurements, peak power represents the energy of the waveform in the selected window, which consists not only of the initial peak but also of the subsequent waveform. Thus peak power may be a more valuable measure than amplitude in determining changes in spinal cord function.

As time-frequency parameters have not been applied to spinal cord monitoring, no criteria have been developed for when a warning should be given. Establishment of these criteria is critical in determining the effectiveness of variables used in detecting potential spinal cord injury. A 50\% decrease in peak power is not equivalent to a $50 \%$ decrease in amplitude, and vice versa, as standard signal power is related to the square of amplitude. Peak power is used in timefrequency analysis because signal energies are represented within a specific time and frequency range, whereas amplitude represents a scalar parameter based on the dominant peak of the time domain waveform. From the results of the present study (table 2), a 30\% decrease in peak power appears to be a rigorous criterion that may provide high sensitivity but low specificity, and could therefore create false positive warnings during surgery. A 70\% decrease in peak power may result in lower sensitivity than either a $50 \%$ or a $30 \%$ decrease, and therefore we recommend a $50 \%$ decrease in peak power as the criterion for intraoperative spinal cord monitoring.

The change in peak frequency may be influenced by interference with similar spectral characteristics as with the SEP signal. The shifts in the peak frequency that occur with injury, and the relative monitoring criteria, should be subject to study in greater detail, as it seems that a $10 \%$ or greater shift in peak frequency may not be an indicator of spinal cord injury. However, these warning criteria for time-frequency variables are simply initial recommendations based on a few clinical cases. It should be noted that the signal to noise ratio is still a limiting factor in applying time-frequency analysis to SEP. SEP recorded with a poor signal to noise ratio may result in a false time-frequency analysis.

There are various algorithms for time-frequency analysis. Short time Fourier transform (STFT), Cohen's class timefrequency analysis, and wavelet transform based timefrequency analysis are all commonly used methods. We used STFT as the prototype in the present study as it is a fundamental method and an extremely powerful tool in many areas. Its interpretation is also easy to understand in both time 
and frequency domains. However, applying STFT to SEP results in poor resolution because of the shorter time course in the waveform analysis. To improve time and frequency resolution, several bilinear transforms are available for consideration and comparison in further investigations.

Time-frequency analysis of SEP signals shows a single peak in a certain time-frequency space, which is easily identified and measured. The maximum power of the selected peak can be computed automatically. Application of time-frequency SEP analysis may decrease false positive monitoring outcomes but the possibility of false negative outcomes may increase. In a previous experimental study, ${ }^{9}$ there was a lower false negative rate in time-frequency analysis of SEP for spinal cord monitoring. However, clinical validation on large series of operated patients is necessary for further confirmation. Timefrequency analysis of SEP could then be a useful and relevant form of intraoperative monitoring to detect possible neurological injury.

\section{Conclusions}

Normal SEP signals appear to occupy a limited proportion of the time-frequency field, and changes in their location have been shown to be useful aids for intraoperative spinal cord monitoring. Peak time was sometimes found to be of too low resolution for practical use in intraoperative spinal cord monitoring, but may be a more useful variable with other types of time-frequency analysis algorithm than STFT. Peak frequency seems to be the major component of the signal. A stable peak frequency should be found in time-frequency analysis of SEP in a particular patient, but the location of this peak will be variable between patients. A shift in peak frequency for a particular patient could, however, be a good predictor of an unreliable trial, a low signal to noise ratio, or spinal cord injury. Peak power of the SEP has been shown to be a more reliable indicator of spinal cord function than amplitude. When monitoring shows an abnormal SEP signal during surgery, SEP peak power could be another reliable way of determining possible neurological damage.

Time-frequency analysis of the SEP seems to have more merit than time domain measurement in monitoring the spinal cord during scoliosis surgery. The application of timefrequency analysis to SEP monitoring allows easy identification of variables that can be shown to be more reliable than those in the time domain. In conclusion, our study showed the value and applicability of time-frequency analysis to SEP monitoring, and its validity as an adjunct to conventional latency-amplitude measurements of SEP to improve the reliability of intraoperative spinal cord monitoring.

\section{ACKNOWLEDGEMENTS}

This study was supported by The Hong Kong Research Grants Council RGC 227/95M and (1995-1998). We wish to thank Dr Andrew Holmes for English editing.

\section{Authors' affiliations}

Y Hu, K D K Luk, W W Lu, J C Y Leong, Department of Orthopaedic Surgery, The University of Hong Kong, Hong Kong

Competing interests: none declared

\section{REFERENCES}

1 Nuwer MR, Dawson EG, Carlson LG, et al. SEP spinal cord monitoring reduces neurologic deficits after scoliosis surgery: results of a large multicenter survey. Electroencephalogr Clin Neurophysiol 1995;96:6-11.

2 Nuwer MR. Spinal cord monitoring with somatosensory techniques. J Clin Neurophysiol 1998;15:183-93.

3 Imai M, Harada Y, Atsuta Y, et al. Automated spinal cord monitoring for spinal surgery. Paraplegia 1989;27:204-11.

4 Nuwer MR. Intraoperative evoked potential monitoring of the spinal cord. A restricted filter, scalp method during Harrington instrumentation for scoliosis. Clin Orthop 1984;183:42-50.

5 Ryan TP, Britt RH. Spinal and cortical somatosensory evoked potential monitoring during corrective spinal surgery with 108 patients. Spine $1986 ; 11: 352-61$

6 Noss RS, Boles CD, Yingling CD. Steady-state analysis of somatosensory evoked potentials. Electroencephalogr Clin Neurophysiol 1996; 100:453-61.

7 Braun JC. Detection of neurological injury using time-frequency analysis of the somatosensory evoked potential. Electroencephalogr Clin Neurophysiol 1996;100:310-18.

8 Thakor NV, Guo XR, Vaz CA, et al. Orthonormal (Fourier and Walsh) models of time-varying evoked potentials in neurological injury. IEEE Trans Biomed Eng 1993;40:213-21.

9 Hu Y, Luk KDK, Lu WW, et al. Prevention of spinal cord injury with time-frequency analysis of evoked potentials: an experimental study. $J$ Neurol Neurosurg Psychiatry 2001;71:732-40

10 de Weerd JP, Kap JI. Spectro-temporal representations and time-varying spectra of evoked potentials. A methodological investigation. Biol Cybern 1981:41:101-17.

11 Hu Y, Luk KDK, Lu WW, et al. Comparison of time-frequency analysis techniques in intraoperative somatosensory evoked potential (SEP) monitoring. Comput Biol Med 2002;32:13-23.

12 Luk KDK, Hu Y, Wong YW, et al. Variability of somatosensory-evoked potentials in different stages of scoliosis surgery. Spine 1999;24: 1799-804.

13 Thakor NV. Adaptive filtering of evoked potentials. IEEE Trans Biomed Eng 1987;34:6-12.

14 Vaz C, Kong X, Thakor N. Adaptive estimation of periodic signals using a Fourier linear combiner. IEEE Trans Signal Proc 1994;42:1-10.

15 Geva AB, Pratt H, Zeevi YY. Multichannel wavelet-type decomposition of evoked potentials: model-based recognition of generator activity. Med Biol Eng Comput 1997;35:40-6.

16 Eisen A, Roberts K, Low $M$, et al. Questions regarding the sequential neural generator theory of the somatosensory evoked potential raised by digital filtering. Electroencephalogr Clin Neurophysiol 1984;59:388-95.

17 Kostyunina MB, Kulikov MA. Frequency characteristics of EEG spectra in the emotions. Neurosci Behav Physiol 1996;26:340-3.

18 Shinomiya K, Okamoto A, Komori $\mathrm{H}$, et al. Prognosticating study for cervical myelopathy using evoked spinal cord potentials. Spine 1990; 15:1053-7.

19 Szalay EA, Carollo JJ, Roach JW. Sensitivity of spinal cord monitoring to intraoperative events. J Pediatr Orthop 1986;6:437-41.

20 Rossini PM, Cracco RQ, Cracco JB, et al. Short latency somatosensory evoked potentials to peroneal nerve stimulation: scalp topography and the effect of different frequency filters. Electroencephalogr Clin Neurophysiol 1981;52:540-52. 